MATEC Web of Conferences 22,01019 (2015)

DOI: $10.1051 /$ matec conf/ 20152201019

(C) Owned by the authors, published by EDP Sciences, 2015

\title{
Research on Technique of Extracting Knowledge from Maintenance Experiences Based on Conceptual Graph
}

\author{
Yanbin Liu, Yanling Qian, Long Wang \& Tengfei Xu \\ Laboratory of Science and Technology on Integrated Logistics Support, National University of Defense Tech- \\ nology, Changsha, Hunan, China
}

\begin{abstract}
Experience knowledge is extremely important in maintenance domain. However, it is difficult to express and extract this kind of knowledge. Conceptual Graph is a new and powerful visual knowledge representation method. This paper proposes one technique based on conceptual graph to extract knowledge from experiences accumulated in maintenance actions. This technique introduces conceptual graph to maintenance domain. With VE distribution pump as an example, the method of conceptual graph is introduced first, and then it is applied to expressing knowledge extracted from experiences accumulated in maintenance actions. Finally, the similarity between graphs of new fault case and base cases is computed, through which maintenance staff could find out the most similar case when new fault appears. The causes and solutions of the most similar case could assist maintenance staff to resolve new faults.
\end{abstract}

Keywords: conceptual graph; maintenance experience; extract knowledge; similarity

\section{INTRODUCTION}

Along with the development of technology, the equipment is becoming increasingly complicated, systemic and intelligential, which puts forward higher request of knowledge in maintenance.

At present, maintenance staff relies on their experience knowledge in repairing. Their knowledge mostly comes from technical manuals, experience imparting and maintenance records, which includes equipment's operational principles, operations of repair tools, fault analysis and fault location, and what's more, planning whole repair process.

If there is no sufficient experience knowledge in maintenance actions, some issues may appear, such as shutdown for long time and performance degradation after repeated disassembly and assembly, even more seriously, safety accidents. As a result, it is required that maintenance staffs especially new ones obtain experience knowledge quickly, systematically and fully.

But it is difficult to express and extract experience knowledge in maintenance domain because the knowledge is unstructured. Firstly, the quantity of knowledge in technical manuals is huge, which brings difficulty to obtain knowledge especially useful knowledge in short time. Secondly experience knowledge is usually handed on by seasoned staff, this process is not systemic enough where experience knowledge leaves out unavoidably, and experience knowledge is recessive, immaterial and nonfigurative in human mind, and it is difficult to pass and apply. Finally, although maintenance records have pertinence to some extent, but it is made without strict rules, which makes the knowledge is superfluous and is not used well.

With the development of computer technology, expressing and extracting knowledge has become a hot topic. Several methods have been put forward, such as Predicate Logic Representation, Semantic Network Representation, Production Representation and Frame Representation. Although these methods could express and extract knowledge, there still are some deficiencies: Predicate Logic Representation has low efficiency and complex reasoning process, and could not express heuristic and imprecise knowledge very well; Semantic Network Representation could not guarantee the logic and availability of reasoning process, and does not fit for judgments and deep knowledge; Production Representation has opaque rules, and is not able to express knowledge in hierarchy, what's more, it has low efficiency in searching; Frame Representation does not fit for expressing process knowledge in maintenance.

The method of conceptual graph was firstly proposed by John F. Sowa in 1984 based on linguistics, psychology and philosophy. This method has advantages as follows:

- Strong expressing ability. Natural language could be translated to conceptual graphs and vice versa, which means this method fits for expressing failures, fault analysis and solutions.

- Vivid form. Conceptual graph has the vivid form of graph. Then maintenance staff could obtain knowledge from conceptual graph in short time.

- The ability of extension. If there are same concept nodes between different conceptual graphs, then graphs could be linked together through the same 
node, which fits for knowledge extension of maintenance experience.

- Simple reasoning. Conceptual graph is based on rules, easy to reason. Therefore knowledge in conceptual graph could be reasoned quickly.

At present, conceptual graph is mainly applied to various aspects of artificial intelligence, like natural language processing, semantic analysis and knowledge acquisition. However it's application in maintenance domain is rare. Based on these advantages of conceptual graph in expressing and extracting knowledge, this paper tries to import the method in maintenance, and to make maintenance process faster and more accurate.

\section{METHOD OF CONCEPTUAL GRAPH}

Conceptual graphs consist of concept nodes, relation nodes and directed cable between nodes. Concept nodes represent concrete or abstract concepts, such as "people"," teacher", "color", "research". Relation nodes indicate the relationship between concepts, like AGNT (agent), OBJ (object), MANR (manner), and STAT (status). For example, "Student Xiao Wang studies English hard", so its conceptual graph is as follows:

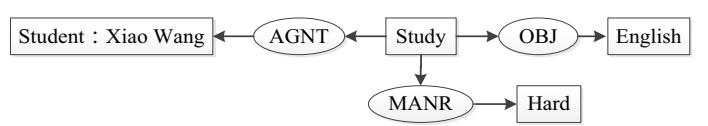

Figure 1. The form of conceptual graph

Where, rectangles indicate concept nodes, oval box indicate relation nodes.
The process of generating conceptual graphs from natural sentences is shown in Figure 2, which includes parsing sentences and extracting their concepts and relations. There are several tools to parse and analyze sentences in Chinese, such as LTP, FNLP, ICTCLAS. Because of the applicability and precision of LTP system, it is chosen to parse sentences and get dependency tree, and thus concepts and relations could be obtained.

Concepts are related to respective domains, which mean different domains have different concepts. Nevertheless relations between concepts could be classified into several types as shown in Table 1.

\begin{tabular}{ll}
\multicolumn{2}{l}{ Table 1. Types of relations } \\
\hline Types of relations & Examples \\
\hline Experience-relation & require, generate \\
Temporal-relation & before, after \\
Usual-relation & agent, object, characterization, \\
Logical-relation & implication, influence \\
Spatial-relation & outside, inside \\
Logic-relation & is, is part \\
\hline
\end{tabular}

\section{ABSTRACT FRAME}

\subsection{Extraction of experience knowledge}

Experience knowledge based on conceptual graph contains three aspects: faults, fault analysis and solutions as is shown in Figure 3, where faults include devices' numbers and fault description while solutions include repair process, maintenance person and repair time.

In the case of VE distribution pump, "It is difficult to start engine" is a common fault. According to repair experience, primary causes of the fault and corre-

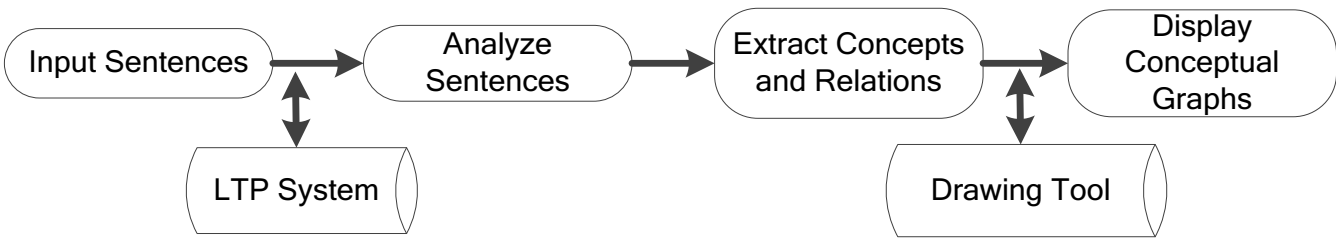

Figure 2. Generation process of conceptual graphs

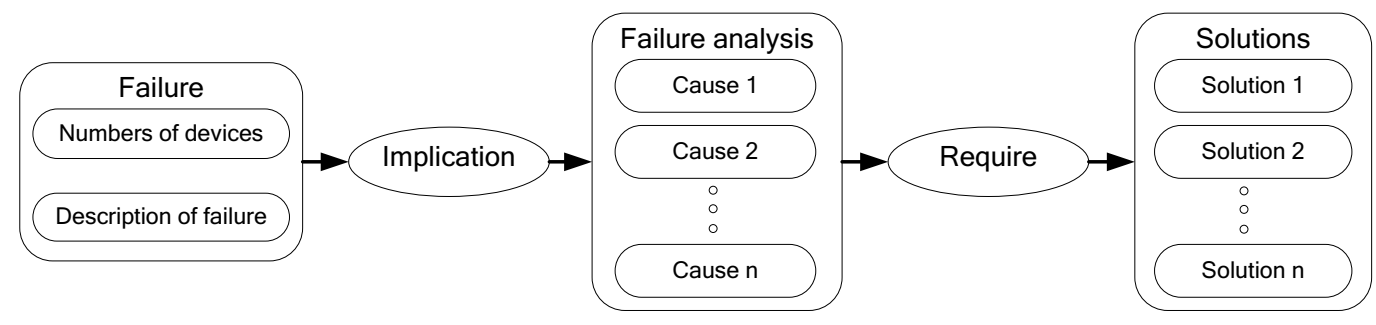

Figure 3. Knowledge expressing in form of conceptual graph 
ICETA 2015

Table 2. Possible causes and solutions of failures

Possible causes

1. The fuel supply advance angle is wrong
2. There is air in fuel system

3. The fuel percolator jams.

4. Solenoid valve stops working

5. Plunger frays or locks.

6.Fuel spray nozzle jams or locks

7. Oil-return screw and oil-inlet screw are installed by contraries.

8. Battery's Voltage is too low

9. Closing plug damages and leaks oil

10. Delivery valve's type is wrong

Solutions

1. Adjust the fuel supply advance angle

2. Remove air

3. Replace the fuel percolator.

4. Replace the solenoid valve

5. Replace the plunger.

6. Wash or replace the fuel spray nozzle.

7. Install oil-return screw and oil-inlet screw again.

8 . Charge or replace the battery.

9. Replace closing plug.

10. Replace delivery Valve

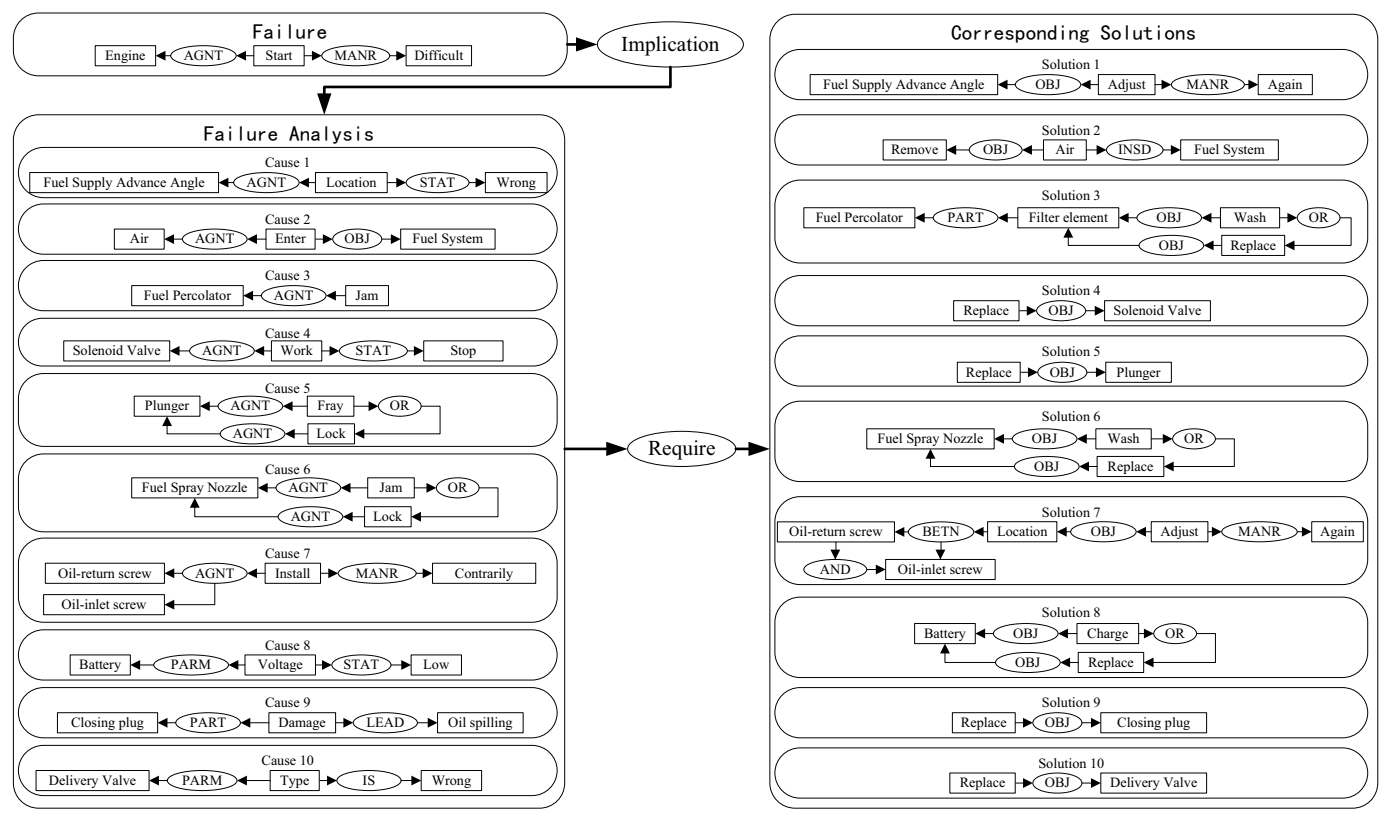

Figure 4. Integrated conceptual graph

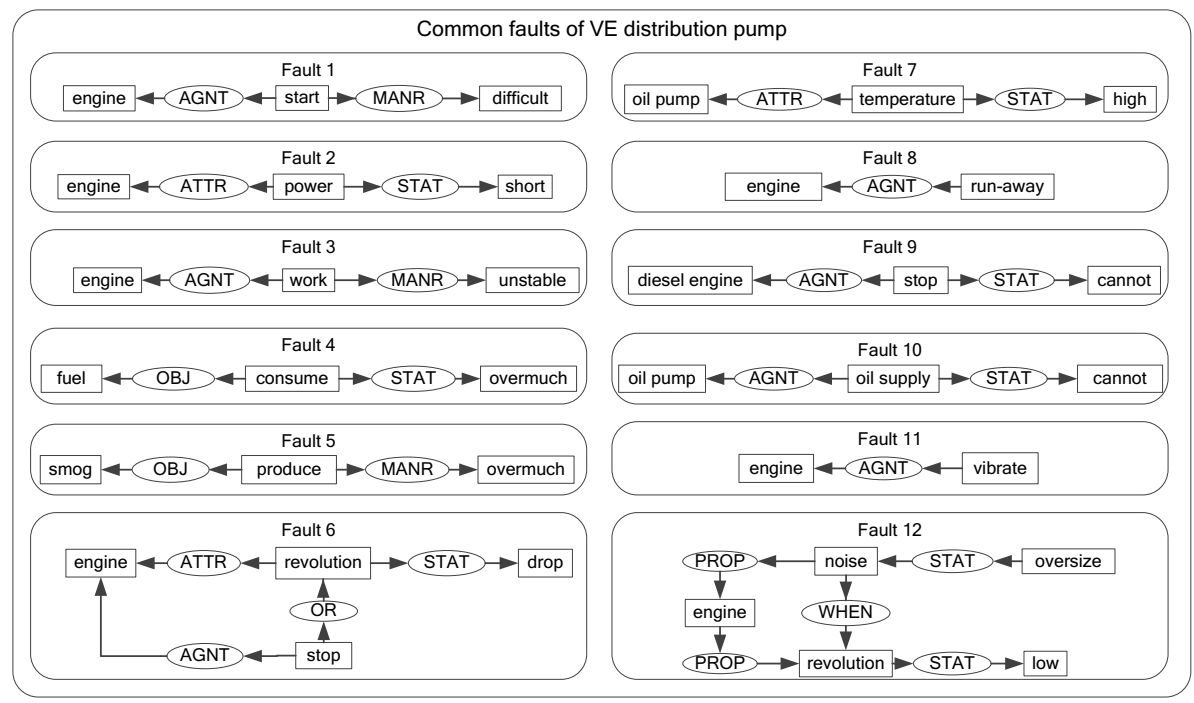

Figure 5. Common failures of VE distribution pumps sponding solutions are shown in Table 2. 
Use conceptual graph to express the content in Table 2, which is shown in Figure 4. We can see, conceptual graph could express the fault "It is difficult to start engine" in vivid form. It is very convenient for maintenance staff to search and reuse.

In addition, the experience knowledge of repairing faults is extracted in the form of conceptual graphs and stored in the database, which facilitates the management of the operation and subsequent reasoning or matching.

\subsection{Reuse of experience knowledge}

Obtained by maintenance records, the conceptual graphs of maintenance experience can be applied to assisting in fault diagnosis and maintenance. This is reuse process of maintenance knowledge where conceptual graphs are reasoned and matched, the similarity between new fault case and existing cases is calculated to determine the most similar case in case base to reason the fault causes and solutions.

The new fault case needs to be expressed in form of conceptual graph, and then reasoning and matching could be carried out. After calculating the similarity between new failure case and the existing cases, and the most similar case in case base could be found, whose cause and solutions may be what wanted to solve the new one. The conceptual graph of common faults of VE distribution pump is shown in Figure 5, if a new fault emerges, it will be matched to these existing cases.

For example, there is a new fault "The engine cannot start", whose conceptual graph is shown in Figure 6. Now this new fault is matched to the first common failure of VE distribution pumps, "It is difficult to start engine", whose conceptual graph is shown in Figure 7.

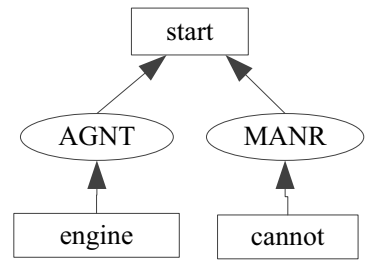

Figure 6. The conceptual graph of "The diesel engine cannot start"

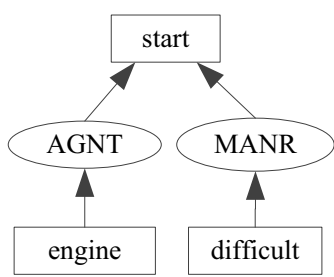

Figure 7. The conceptual graph of "It is difficult to start engine"

Calculating the similarity of two conceptual graphs requires calculating the similarity of concepts and relations first, and summating similarity of concepts and relations with weight. Each conceptual graph has an entrance, which points out the core concept of the graph and the key point to calculate the similarity of concept graphs.

Similarity calculation between concepts is based on the structural organization of concepts in HowNet. Because of different hyponymy and synonymy in conceptual system, concept similarity is calculated according to conceptual distance. For two concepts of $c_{1}$ and $c_{2}$, whose concept distance is $\operatorname{dist}_{c}\left(c_{1}, c_{2}\right)$, the similarity between them is:

$$
\operatorname{sim}\left(c_{1}, c_{2}\right)=\frac{\alpha}{\operatorname{dist}_{c}\left(c_{1}, c_{2}\right)+\alpha}
$$

Where, $\alpha$ is a regulative parameter, and is the distance value when the concept similarity is 0.5 .

The similarity between relations could be simplified into whether the two relations are same, if it is, then the similarity is 1 , otherwise, 0 . Then relation similarity between $r_{1}$ and $r_{2}$ is:

$\operatorname{sim}\left(r_{1}, r_{2}\right)=\left\{\begin{array}{l}1, r_{1}=r_{2} \\ 0, r_{1} \neq r_{2}\end{array}\right.$

Except for determining the concept similarity and relation similarity, different weight for different nodes should be added to match conceptual graphs, which could improve precision. The child nodes of relation nodes who have more related nodes have more weight, the method to allocate weight is as followed:

$$
w\left(x_{i}\right)=\frac{n_{i}}{\sum_{t=1}^{t=m} n_{t}^{2}}(1-\beta)
$$

The weight of relation nodes is the number of its brother nodes divided by the summation of the square number of the child number of its brother nodes of the relation. This way to add weight to different nodes insures that the general weight is 1 , and that the similarity is between 0 and 1 .

The root node in Figure 6 is "start", which has two relation nodes: "AGNT" and "MANR". The two relation nodes have one child node separately: "diesel engine" and "cannot". Set the weight of entrance node "start" for $\beta=0.4$, then

$w(\mathrm{i})=\frac{1}{(1+1)}(1-0.4)=0.3$ 


$$
w(\mathrm{j})=\frac{1}{(1+1)}(1-0.4)=0.3
$$

After getting the similarity of concepts and relations, the formula for conceptual graphs' similarity calculation is:

$$
\begin{aligned}
& \operatorname{sim}_{G}\left(c_{1}, c_{2}\right)=w\left(c_{1}, c\right) * \operatorname{sim}_{c}\left(c_{1}, c_{2}\right) \\
& +\sum_{s} w\left(c_{1}, s\right) * \max \left[\operatorname{sim}_{r}\left(r_{1}^{s}, r_{2}^{s}\right) * \operatorname{sim}_{G}\left(c_{1}^{r_{1}^{s}}, c_{2}^{r_{2}^{s}}\right)\right]
\end{aligned}
$$

Where, $\operatorname{sim}_{\mathrm{G}}$ denotes the similarity of conceptual graphs, $\operatorname{sim}_{c}$ denotes the similarity of concepts, and $\operatorname{sim}_{r}$ denotes the similarity of relations; cland $\mathrm{c} 2$ represents the entrance points of two conceptual graphs respectively; $r_{1}^{s}\left(r_{2}^{s}\right)$ denotes the $s^{\text {th }}$ relation of first(second) graph, while $c_{1}^{r_{1}}\left(c_{2}^{r_{2}}\right)$ denotes the entrance point of child graph related to $r^{s}\left(r^{s}\right)$; $w(c, c)$ denotes the weight of entrance, while $w\left(c_{1}, s\right)$ denotes the weight of $s^{\text {th }}$ relation related to entrance. The formula for similarity calculation between Figure 6 and Figure 7 is:

$$
\begin{aligned}
\operatorname{sim}_{G}\left(c_{1}, c_{2}\right) & =w\left(c_{1}, c\right) * \operatorname{sim}_{c}(\text { "start", "start") } \\
& +w\left(c_{1}, 1\right) * \operatorname{sim}_{r}(\text { AGNT, AGNT }) \\
& * \operatorname{sim}_{c}(" \operatorname{diesel} \text { engine", " engine") } \\
& +w\left(c_{1}, 2\right) * \operatorname{sim}_{r}(\text { MANR, MANR }) \\
& * \operatorname{sim}_{c}\left(" \text { cannot" }^{\prime}, \text { difficult" }\right)
\end{aligned}
$$

Where, $\quad w(c, c)=0.4 ; w(c, 1)=w(c, 2)=0.3 ;$ and $\operatorname{sim}_{r}($ AGNT, AGNT $)=\operatorname{sim}_{r}($ MANR, MANR $)=1$.

Using the tool based on HowNet to calculate concept similarity:

$\operatorname{sim}_{c}($ " engine", " engine") $=1$

$\operatorname{sim}_{C}($ " cannot", "difficult" $)=0.73508$

The similarity between Figure 6 and Figure 7 is:

$$
\begin{aligned}
\operatorname{sim}_{G}\left(c_{1}, c_{2}\right) & =0.4 \times 1+0.3 \times 1+0.3 \times 0.7351 \\
& =0.92053
\end{aligned}
$$

In this way, similarity between common faults and new fault could be calculated. In this way, when new fault appears maintenance staff could find out the most similar case, whose analysis and solutions could be referred to the new one. Furthermore, all fault cases could be stored in a database, and with maintenance actions going on, the number of cases in database will increase. When the number is enormous enough, most fault cases could be searched in the database, which would be a powerful tool to maintenance.

\section{PRACTICAL APPLICATION}

In practical application, we have found twelve common faults of VE distribution pumps according to the experience of a maintenance department. Simultaneously, these faults' possible causes and corresponding solutions are obtained. Then a case base is built containing these source cases.

Now a faulty engine waits to be diagnosed and repaired. The engine cannot start even though it has plenty fuel. After primary check, the fault is locked in the VE distribution pump.

The proposed technique is applied to fault diagnosis. At first the conceptual graph of new fault "the engine cannot start" is confirmed, which is shown in Figure 6. Then the similarity between the new fault and the source cases is calculated using proposed arithmetic and the result is shown in Table 3 . Finally the most similar case would be found from the result.

Table 3. Similarity between new fault and source cases

\begin{tabular}{l|l}
\hline Faults & Similarity \\
\hline Fault 1 & 0.92053 \\
Fault 2 & 0.01778 \\
Fault 3 & 0.49108 \\
Fault 4 & 0.14819 \\
Fault 5 & 0.11000 \\
Fault 6 & 0.36038 \\
Fault 7 & 0.02824 \\
Fault 8 & 0.36038 \\
Fault 9 & 0.66038 \\
Fault 10 & 0.52547 \\
Fault 11 & 0.33623 \\
Fault 12 & 0.01778 \\
\hline
\end{tabular}

From Table 3 we can see, different fault case has different similarity where the similarity between new fault and Fault 1 is the largest. Apparently, after getting the result maintenance staff would take Fault 1 into account during fault diagnosis.

Because the largest value is over $90 \%$, it is should be realized that these two cases are similar to a great extent. Figure 4 is the conceptual graph of Fault 1. There are ten possible causes according to previous experiences. Since these two faults are similar so much, the ten causes may possibly appear. These implied causes which lead to Fault 1 should be checked firstly.

According to Figure 4, fuel percolator, solenoid valve, plunger, fuel spray nozzle, battery, closing plug and delivery valve of the VE distribution pump may damage and need repairing or replacing. Maintenance staff is supposed to examine the equipment or parts, and when the fault is located, what the staff needs to do is following the corresponding solutions in Figure 4.

After examining, the staff finds that the plunger has worn badly. Therefore the VE distribution pump could not provide required high-pressure-oil to engine. That's why the engine cannot start. By understanding the working environment and condition of the engine, 
it is realized that the engine has been consuming poor quality fuel in which water and other impurity is too much. In addition, the fuel percolator needs replacing, since it could not stop the impurity in fuel.

The staff replaces the plunger and fuel percolator, assemble the engine, and try to start it. The engine starts normally and returns to original good condition.

From this practical application, we could see that experience knowledge could be extracted in form of conceptual graphs, which provide a path to search the most similar fault case. The causes and solutions of the case could assist maintenance staff to resolve new faults.

The idea of applying conceptual graphs in maintenance domain is proposed recently, and it gives good prospect. In the future, more and more fault cases would be accumulated and the case base would be great enough to assist maintenance staff absolutely.

\section{SUMMARY}

This paper proposes one technique based on conceptual graph to extract knowledge from experiences accumulated in maintenance actions. The method of conceptual graph is first introduced, and then it is used to express knowledge extracted from experiences accumulated in maintenance actions. Finally, the similarity between new fault case and base cases is computed, through which maintenance staff could find out the most similar failure case when new fault appears to reason the possible causes and find effective solution.

However the calculation of concept similarity is based on HowNet whose content is enormous and the part which is related to maintenance is so small that the result is on the low side. In the future, research on limiting semantic bound in some special domains should be carried on, and then the concept similarity would be more accurate.

Although the method of conceptual graph is applied to maintenance domain at the initial stage, a good application prospect has been shown. The next step, the generation technique of conceptual graphs from fault cases and calculation of similarity should be studied to make the extraction process of experience knowledge in maintenance more quickly and accurately. In addition, we will try to achieve the fault database and searching system, and apply this technique in actual maintenance work.

\section{REFERENCES}

[1] Meihua Li, Daming Han \& Huaimin Lu. 2006. Artificial intelligence applied in machinery fault diagnosis. Forest Engineering, 3: 22.

[2] Bernard Kamsu-Foguem, Gayo Diallo \& Clovis Foguem. 2013. Conceptual graph-based knowledge representation for supporting reasoning in African tradition- al medicine. Engineering Applications of Artificial Intelligence, 26: 1348-1365.

[3] Miaomiao Zhu \& Guofeng Zhu. 2012. The research and application of knowledge lattice. Science \& Technology Vision, 28: 172-173.

[4] B. Kamsu Foguem, T. Coudert, L. Geneste \& C. Beler. 2008. Knowledge formalization in experience feedback processes: An ontology-based approach. Computers in Industry, 59: 694-710

[5] Xiaoguang Yue \& Xiaocheng Liang. 2010. Chinese Word Segmentation System Design and Implementation Based on .NET. Microcomputer Information.

[6] Qun Liu \& Sujian Li. 2002. Word Similarity Computing Based on How-net. CLSW2002: 67-76. Taipei.

[7] Bernard Kamsu-Foguem \& Daniel Noyes. 2013. Graph-based reasoning in collaborative knowledge management for industrial maintenance. Computers in Industry, 64: 998-1013.

[8] Xiaoluan Zhang \& Xifeng Wang. 2011. Study and implementation of Chinese semantic computation based on conceptual graph. Computer Engineering and Applications, 47: 120-123.

[9] Siti Sakira Kamaruddin, Abdul Razak Hamdan, Azuraliza Abu Bakar \& Fauzias Mat Nor. 2009. Dissimilarity algorithm on conceptual graphs to mine text outliers. 2009 2nd Conference on Data Mining and Optimization. Malaysia: Selangor.

[10]Paula Andrea Potes Ruiz, Bernard Kamsu-Foguem \& Daniel Noyes. 2013. Knowledge reuse integrating the collaboration from experts in industrial maintenance management. Knowledge-Based Systems, 50: 171-186.

[11]A. Seguy, D. Noyes \& P. Clermont. 2010. Characterisation of collaborative decision making processes. International. Journal of Computer Integrated Manufacturing, 23: 1046-1058. 